\title{
Adoptive T-Cell Therapy of Melanoma: Promises and Challenges
}

\author{
Patrick Schmidt and Hinrich Abken \\ Centre for Molecular Medicine Cologne and Clinic I for Internal Medicine, \\ University of Cologne, Cologne \\ Germany
}

\section{Introduction}

Adoptive immunotherapy for the treatment of melanoma has attracted growing interest in recent years. The trend reflects, at least in part, the disappointing results of conventional chemotherapy to induce lasting remission in advanced stages of the disease with multiple metastases and to cure patients even after initially complete response. The isolation of tumor infiltrating $\mathrm{T}$ cells from melanoma lesions, the identification of melanoma-associated antigens and the significant progress in our understanding in redirecting an immune response favored the development of novel strategies in adoptive immunotherapy of melanoma. Most recent advances in selectively eliminating melanoma cell subsets from tumor tissues together with the identification of so-called melanoma stem cells, however, imply to re-define the therapeutic target in melanoma. The review discusses current challenges and perspectives in the rational design of cellular immunotherapy of melanoma.

\section{The need for novel options in the treatment of advanced stage melanoma}

Despite of major improvements in the therapy of melanoma, the frequency of partial or complete responses after chemo- and radiotherapy has not significantly risen since years (Garbe et al. 2010). Disseminated melanoma is still an incurable disease and overall survival correlates with the stage of the disease at time of diagnosis. A 10-year-survival rate of 75$85 \%$ can be reached when the primary tumor was diagnosed in stage I or II, melanoma in stage III or IV, however, is commonly associated with poor survival (Garbe et al. 2010). The situation is mainly caused by two major drawbacks due to the particular biology of melanoma cells. Firstly, melanoma early disseminates into distant organs including the brain by forming micro-metastases which are small in cancer cell numbers and beyond the detection limit of current tomographic procedures (Denninghoff et al. 2004; Bedikian et al. 2010). Micro-metastases can persist for long time without any change in size in a "dormant" stage (Leiter et al. 2010). Secondly, many melanoma cells are notoriously resistant against chemo- and radiation therapy (Bradbury \& Middleton 2004; Pak et al. 2001; Pak et al. 2004). Both drawbacks result in the unsatisfactory situation that surgical removing of tumor lesions in early stages is the only curative option to fight melanoma. In more progressed stages of the disease recruitment of the cellular immune defense is thought to be an option. In this context immune-modulatory adjuvants, including interferon $a-2 b$, exhibit some effect 
although are not curative (Kirkwood et al. 2000; Kirkwood et al. 1996) and still a matter of debate. These efforts, however, indicate that activation of the patient's immune response can be effective in fighting melanoma; a more tumor-specific way of immune cell activation needs to be explored. We here summarize evidence for the potency of adoptive immune cell therapy in melanoma and discuss premises and future treatment options.

\section{Adoptively transferred T cells are capable to fight melanoma}

Based on early evidences that the concerted action of the immune system is capable to fight cancer efforts were focused to redirect cytolytic effector cells towards autologous cancer cells. First experiments following the strategy were performed by the Rosenberg group in 1984 by treating transplanted tumors in mice by adoptive transfer of syngeneic lymphocytes in combination with IL-2 (Rosenberg 1984; Grimm et al. 1982). In addition to promote activation and proliferation of both cytolytic and helper T cells, IL-2 also activates natural killer (NK) cells as well as macrophages (Waldmann \& Tagaya 1999) which provided the rationale to administer IL-2 as immune adjuvant in cancer patients. Between 1985 and 1993 overall 270 melanoma patients were treated with IL-2 in the context of eight clinical trials with an objective response rate of $16 \%$, comprising $6 \%$ complete and $10 \%$ partial responses (Atkins et al. 1999). While IL-2 doubtless induced an immune cell anti-tumor response, the immune cell activation was not specifically redirected towards melanoma.

The elucidation of so-called "tumor antigens" preferentially expressed on melanoma cells led to the concept to immunize patients with defined $\mathrm{T}$ cell antigens in order to activate by natural selection melanoma specific $\mathrm{T}$ cells in a more specific fashion. Two antigens were primarily considered as promising candidates, namely Melan-A/MART 1 and gp100 (Rivoltini et al. 1996; Rivoltini et al. 1995). By using different strategies of vaccination including injection of antigen or application of antigen-pulsed dendritic cells melanoma reactive $T$ cells could be amplified in the cancer patient (Kawakami \& Rosenberg 1997). Until today a variety of clinical studies using dendritic cells loaded with $\mathrm{T}$ cell recognition peptides were performed with various therapeutic effects. A meta-analysis in 2008 comprising 38 trials with overall 626 patients revealed that $3 \%$ of the patients responded to treatment with complete and $6 \%$ with partial remission, $21 \%$ achieved stable disease (Engell-Noerregaard et al. 2009). These results were irrespective of both the antigen used for loading and the type of DCs. The route of injection and the administration of adjuvant had no impact on clinical outcome. In conclusion no therapeutic advantage could be documented for patients treated with vaccines compared to treatment with immune stimulatory cytokines.

As a consequence further developments in the immunotherapy of melanoma were thereon focused on the adoptive transfer of immune effector cells itself. The development was further strengthened by the success in isolating tumor infiltrating lymphocytes (TILs) which are found in substantial numbers in a variety of melanoma lesions. First described in 1969 (Clark et al. 1969) TILs particularly from melanoma gained interest in during the last years since these cells are believed to recognize melanoma-associated antigens and thereby specifically accumulate in melanoma lesions. Freshly isolated from melanoma lesions TILs are mainly of $\mathrm{T}$ cell origin consisting of both effector and helper $\mathrm{T}$ cell subsets. While the prevalence of TILs in primary melanoma lesions and metastases is not a prognostic factor itself, high numbers of infiltrating lymphocytes, however, correlate with better clinical outcome (Clemente et al. 1996; Burton et al. 2011), moreover providing a rationale to use 
these lymphocytes as effector cells in the treatment of melanoma. Protocols according to GMP standards were developed to isolate TILs and to amplify them ex vivo to numbers suitable for re-infusion (Figure 1). To expand preferentially highly melanoma reactive T cells first lymphocyte expansion protocols were based on cultures in presence of IL-2 on feeder layer cells expressing melanoma antigens (Vignard et al. 2005). While the concept perfectly worked in mouse models, the application in patients turned out to need further improvements. Upon adoptive TIL infusions regression of metastases could be observed in the majority of patients, only few, however, remained in complete remission (Besser et al. 2010). In most cases a partial shrinkage of the tumor mass followed by stable disease was observed. The disappointing results despite the high numbers of infused TILs is thought to be due to the fact that the cells were extensively amplified over weeks ex vivo and have entered anergy unable to be properly rescued for an anti-melanoma attack after infusion (Chhabra 2010). More recent expansion protocols therefore aim to select so-called "young TILs" which underwent minimal culture times and which were not selected for melanoma reactivity prior re-infusion. A first phase I trial showed improved response with increased frequencies in partial and complete remission (Besser et al. 2009). All trials were performed under myeloablative conditions and IL-2 administration based on the rationale to provide space and stimuli for homeostatic expansion of adoptively transferred $T$ cells. Other cytokines like IL-7 and/or IL-15 are worth to be explored since they do not activate regulatory $\mathrm{T}$ cells.

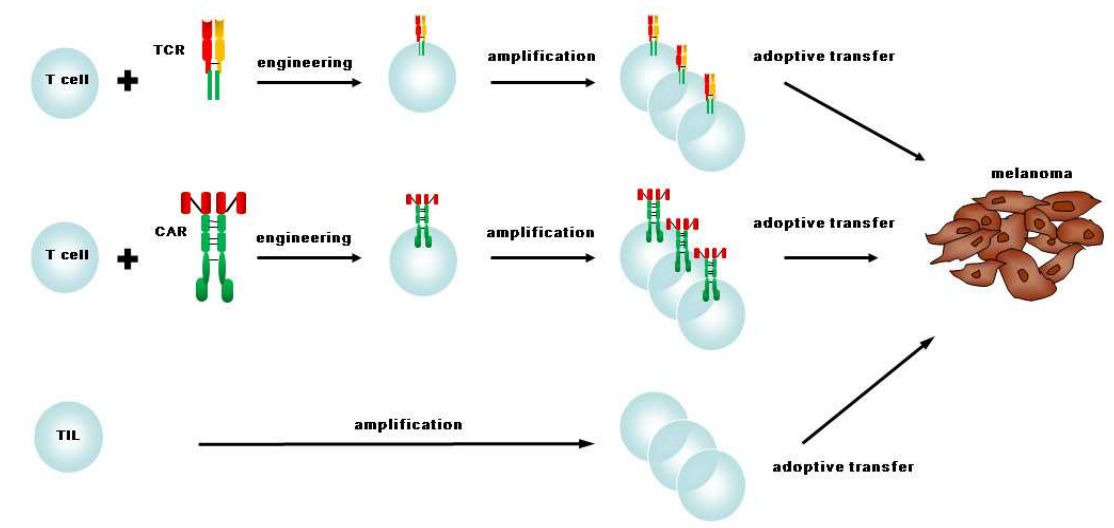

Fig. 1. Strategies in the adoptive immunotherapy of melanoma.

Tumor infiltrating lymphocytes (TIL) are isolated from a melanoma lesion, amplified ex vivo and re-administered without further modification. Alternatively, $\mathrm{T}$ cells from the peripheral blood of the melanoma patient are engineered ex vivo to express either a chimeric antigen receptor (CAR), which forms a homo-dimer, or a recombinant $\mathrm{T}$ cell receptor (TCR), which is composed of the a and $\beta$ chain, with specificity for a melanoma associated antigen, amplified and re-administered in therapeutic doses to the patient.

\section{Redirecting $T$ cells with pre-defined specificity towards melanoma}

While adoptive transfer of TILs is showing promising efficacy in first trials, attempts are made to redirect blood $\mathrm{T}$ cells with pre-defined specificity toward melanoma. The rationale 
for using effector $\mathrm{T}$ cells from the peripheral blood is provided by the frequent inability to isolate TILs in sufficient amounts from a given melanoma lesion and the more progressed proliferative stage of TILs compared to blood T cells.

To redirect peripheral blood $\mathrm{T}$ cells specifically towards melanoma antibodies were combined in a bi-specific antibody format which targets $\mathrm{T}$ cells with by an anti-CD3 antibody as one arm and the melanoma cell with the other arm. Upon application it is assumed that bi-specific antibodies build a bridge between $\mathrm{T}$ cells and melanoma cells thereby forcing peripheral blood cytolytic effectors towards their target. Bi-specific antibodies were directed against melanoma p97, gp100 (Cochlovius et al. 1999), and melanoma chondroitin sulfate proteoglycan (MCSP, also called high molecular weightmelanoma associated antigen HMW-MAA) (Grosse-Hovest et al. 1999). Therapeutic potency was demonstrated by long-term survival in pre-clinical models resulting, the efficacy, however, critically depends on the number of recruitable effector cells at the tumor site. Bispecific constructs triggering CD28 costimulation of T cells furthermore increased tumor-cell killing predominantly by recruiting cytolytic cells without targeted specificity (GrosseHovest et al. 1999; Grosse-Hovest et al. 2003).

While first generation bi-specific reagents were conventional hetero-dimeric antibodies, second generation antibodies are constituted of two single polypeptide chain antibodies covalently joined by a linker domain (Grosse-Hovest et al. 1999; Bluemel et al. 2010a). Those so-called bi-specific T cell engager (BiTE) antibodies targeting CD3 on T cells and a tumorassociated antigen on malignant cells of solid tumors showed efficacy even in nano-molar concentrations in a clinical trial (Bargou et al. 2008). The size of antigen as well as the targeted epitope impacts the efficacy in T cell activation since a BiTE antibody targeting the membrane proximal domain of HMW-MAA on melanoma cells showed more potent than those binding to more distal domains (Bluemel et al. 2010b). The same observations were made when targeting EpCAM indicating that there is obviously a correlation between epitope positioning relative to the cell membrane and the potency in $\mathrm{T}$ cell activation.

Technical advances in genetically engineering blood T cells ex vivo fueled efforts to design $T$ cells with pre-defined specificity for redirected targeting melanoma cells. Recombinant DNA technology and the structural elucidation of the T cell receptor (TCR) complex together with recent developments in vector design made the genetic $\mathrm{T}$ cell modification with a recombinant TCR feasible. The melanoma gp100 specific TCR was one of the first be cloned from $\mathrm{T}$ cells accumulating at the tumor site. TCR $\alpha$ and $\beta$ chains were introduced ex vivo by retroviral gene transfer into naïve, fresh $T$ cells which gained specificity as indicated by redirected cytotoxicity towards primary melanoma and established melanoma cell lines and by increased secretion of pro-inflammatory cytokines including IFN- $\gamma$ (Schaft et al. 2003; Morgan et al. 2003). TCR modified T cells were amplified ex vivo and re-infused in therapeutic doses to the patient (Figure 1). Similarly, recombinant TCRs with specificity for MART1/Melan-A or MAGE-A1 could be transferred into naïve T cells (Hughes et al. 2005; Willemsen et al. 2005).

In 2006 the Rosenberg group published the first clinical trial using $\mathrm{T}$ cells with engineered specificity for melanoma. Adoptive transfer of those $\mathrm{T}$ cells caused a strong response against metastases in distant organs. Engineered $\mathrm{T}$ cells persisted in circulation of most patients for nearly 2 months. The therapeutic efficacy, however, was disappointingly low with 2 out of 17 patients showing objective regression of metastases resulting in complete response. In those patients, interestingly, engineered $\mathrm{T}$ cells were detected in the blood for a year after treatment implying that therapeutic efficacy may correlate with long-term anti-melanoma 
immunity (Morgan et al. 2006; Coccoris et al. 2008). In a very recent trial, similarly TCR engineered $\mathrm{T}$ cells showed efficacy towards brain metastases of melanoma indicating that this procedure may be useful to treat otherwise incurable metastatic sites (Hong et al. 2010). Although these and other trials showed technical feasibility in engineering TCR modified T cells and clinical practicability in the adoptive transfer of those cells, the unexpected low clinical response raised concerns with respect to clonal variability of the targeted melanoma lesion.

Melanoma like other malignant diseases displays clonal evolution during tumor progression which enables the tumor to evade $\mathrm{T}$ cell recognition. Common mechanisms are the downregulation of MHC complex expression (Seliger 2008), point mutations in the $\beta_{2}$ microglobulin chain (Sigalotti et al. 2004), and deregulation of various components of the antigen processing machinery (Seliger 2008; Vitale et al. 2005). As a consequence TCR engineered $\mathrm{T}$ cells are no longer able to recognize and destroy those melanoma cells with mutant or lack of MHC-peptide complexes.

Limitations in the expression of a recombinant TCR in T cells additionally dampened enthusiasm in the strategy. Co-expression of a recombinant TCR together with the physiological TCR in T cells turned out to create new but unpredictable specificities which may result in severe auto-reactivity. The molecular basis is the similarity of the recombinant $\alpha$ and $\beta$ TCR chains to the respective chains of the physiological TCR which leads to the formation of hetero-dimers loosing the specificity of the recombinant TCR (Coccoris et al. 2010). In mouse models hetero-dimer formation resulted in severe auto-immunity (Bendle et al. 2010). Tremendous efforts were undertaken to solve the problem including replacement of TCR constant domains by homologous murine domains (Cohen et al. 2006) and introducing cysteine bridges (Kuball et al. 2007) to enforce $\alpha \beta$-pairing of the recombinant TCR chains.

Advances in the engineering of recombinant signaling molecules facilitated the development of a "one-chain-receptor" molecule for redirected $\mathrm{T}$ cell activation. Zelig Eshhar pioneered the strategy in generating a chimeric antigen receptor (CAR) molecule composed of an extracellular single chain antibody for binding and an intracellular TCR signaling domain (Eshhar et al. 1993). The CAR, also named immunoreceptor or nick-named "T-body", thereby combines the MHC-independent recognition of antigen by an antibody with the T cell activating machinery of the TCR. Naïve T cells from the peripheral blood of melanoma patients are engineered ex vivo to express the CAR, amplified and administered to the patient (Figure 1). Due to the modular design a nearly unlimited variety of antigens can be targeted as long as an antibody is available, including non-classical $\mathrm{T}$ cell antigens like carbohydrates as HMW-MAA, also called MCSP (Reinhold et al. 1999). Different CARs were reported to target melanoma antigens, including HMW-MAA (Reinhold et al. 1999), melanotransferrin (Schmidt et al. 2011), GD2 (Yvon et al. 2009) and GD3 (Lo et al. 2010). The modular composition of CARs moreover allowed combining primary signaling moieties with costimulatory signals in order to modulate the $\mathrm{T}$ cell response in a predicted fashion (Hombach \& Abken 2007). Along with multiple other modifications the stability of expression and antigen binding has been substantially improved during the last years (Bridgeman et al. 2010). Although CARs use the TCR signaling machinery, the strategy is obviously not restricted to redirect T cells; monocytes, macrophages as well as NK cells can be specifically redirected by CARs as well (Pegram et al. 2008; Kruschinski et al. 2008). Appropriate models and, finally, trials need to address whether redirected non-T cells have a benefit in tumor elimination in general and in melanoma therapy in particular. 
Some TCRs and CARs specific to melanoma associated antigens are already applied in phase I clinical trials, and several others are in an advanced phase of preparation (Table 1). Only a few of these studies have been published; other information is derived from registries and from personal communications.

A clinical trial by the Brenner group, Baylor College of Medicine, (Pule et al. 2008) has shown a correlation between the persistence of adoptively transferred, modified $\mathrm{T}$ cells and the clinical outcome. Epstein-Barr virus specific cytotoxic T cells were engineered to express a CAR directed to the diasialoganglioside GD2, an antigen expressed by human neuroblastoma cells, aiming that redirected $\mathrm{T}$ cells would receive optimal costimulation after engagement of their EBV-specific TCR, enhancing survival and anti-tumor activity mediated through their CAR (Savoldo et al. 2007). When administered to tumor patients the EBV-specific $T$ cells engineered with a GD2-specific CAR indeed survived longer than T cells with the same CAR but lacking virus specificity. Infusion of these genetically modified $\mathrm{T}$ cells was associated with tumor regression or necrosis in half of the patients. Results show the safety, feasibility, and potential anti-tumor activity of adoptive therapy with CAR modified T cells; clinical application, however, has to take into account some particular aspects as recently summarized (Büning et al. 2010) and briefly discussed below.

\section{Advantages, premises and challenges of engineered T cells in their attack against melanoma}

There are potential balances to advantages of the CAR or the TCR strategy in the adoptive T cell therapy of melanoma which need careful consideration. Melanoma cells frequently down-regulate proteins associated with antigen processing and presentation (Seliger et al. 1997) which effectively renders the tumor cell invisible to a physiological $T$ cell attack. Consequently, the MHC-independent targeting of cell surface molecules through a CAR makes those melanoma cells vulnerable to $\mathrm{T}$ cell attack while remaining invisible for TCR engineered T cells. The CAR strategy moreover has the advantage that immune cells with defined specificity for a variety of cell surface molecules can be produced. Powerful selection systems, such as phage display provide a plethora of binding domains to target virtually any cell surface molecule. Melanoma cells which do not express the targeted antigen, however, are not attacked by CAR redirected $\mathrm{T}$ cells making antigen-loss cell variants a source of potential tumour relapse after initially successful treatment. TCR redirected $\mathrm{T}$ cells, on the other hand, may recognize even those melanoma cells when they cross-present the targeted antigen. Most recently, TCR-like single chain antibodies were generated and used as targeting domain in a CAR, thereby combining the MHC-restricted recognition of antigen with the CAR strategy. T cells with TCR-like CAR were redirected towards NY-ESO-1 and MAGE-A1, respectively (Stewart-Jones et al. 2009; Willemsen et al. 2001). The advantage of these MHC restricted CARs over the use of recombinant TCRs remains unclear aside from the fact that the CAR consists of a single expressed protein while the TCR approach requires co-expression of both $\alpha$ and $\beta$ TCR chains.

The high complexity of the recognition and signaling process in the $\mathrm{T}$ cell and the variety of antigen structures on the melanoma cell imply that the optimized configuration of a CAR universal for each antigen may not exist. In contrast the TCR recognition and activation process through MHC presented antigen is much more standardized. The antibody-derived binding domain of a CAR moreover displays extraordinary high affinity compared to a TCR. By mutagenesis the affinity of a given antibody binding domain can be increased or 
reduced (Stewart-Jones et al. 2009; Chmielewski et al. 2004). Increase in affinity, however, does not necessarily improve CAR redirected $\mathrm{T}$ cell activation which is assumed for TCR mediated $\mathrm{T}$ cell activation as well. CD28 costimulation, moreover, does not impact the activation threshold of CAR redirected T cells (Chmielewski et al. 2011).

Critical to the success of therapy will moreover be the choice of targeted antigen and the membrane topography of the targeted epitope. In vitro data suggest that an optimal $\mathrm{T}$ cellto-target cell spacing distance is required for T cell activation. For instance, binding the more membrane distal epitope of the targeted antigen showed less $\mathrm{T}$ cell activation than binding of the membrane proximal epitope (Hombach et al. 2007). The rigidity of the antigen itself as well as the antigen mobility in the cell membrane will moreover impact redirected $\mathrm{T}$ cell activation. Carbohydrate antigens like HMW-MAA are thought to act differently than polypeptide antigens like melanotransferin, both expressed on the same melanoma cell. As such, empirical testing may be required to identify the best targeted epitope for the particular antigen for redirected $\mathrm{T}$ cell activation.

The TCR or CAR binding avidity impacts recruitment of engineered T cells to tumor sites. Strong binding to target antigen may cause the $\mathrm{T}$ cells to be locally trapped while low avidity interactions may not provide sufficiently long $\mathrm{T}$ cell - melanoma cell contact to execute the cytolytic attack. On the other hand, the amount of antigen on the melanoma cell surface is also important. In essence, low affinity binding directs the activity of engineered T cells preferentially against targets with abundant antigen levels; high affinity binding is thought to be also effective against low antigen levels on target cells. The optimized affinity which sustains selective T cell trafficking to the tumor, however, is still unclear. Homing and migration of engineered $\mathrm{T}$ cells may also be manipulated through co-expression of chemokine receptors alongside with CARs (Cheadle et al. 2007). Alternatively, isolated T cell subpopulations which express or lack certain chemokine receptors may be used, in particular when targeting melanoma lesions in the periphery. Studies investigating these issues in animal models are now gaining momentum and are likely to improve the antitumor efficacy. Once entered the tumor lesion engineered $\mathrm{T}$ cells are assumed to efficiently re-cycle lytic capacity and to kill multiple targets. While this is suggested by in vitro evidences formal in vivo confirmation is still lacking.

A beneficial effector cell-to-target cell ratio at the tumor site is likely to be required for efficient target cell lysis. Higher numbers of engineered T cells applied per dose are likely to increase clinical efficacy, an estimation based on clinical data, however, is not yet available. Current trials are applying up to $10^{10}$ cells per dose. These and higher numbers of engineered $\mathrm{T}$ cells can be generated by current expansion protocols; cells with the optimal phenotype for adoptive transfer, however, may not be generated under these conditions. Short term amplification protocols are therefore discussed for both TILs from melanoma lesions and for engineered T cells.

Auto-immunity may result from off-target $\mathrm{T}$ cell activation against healthy tissues which physiologically express low levels of the targeted antigen. A number of so-called "tumorassociated antigens" are also expressed on healthy tissues, although frequently at lower levels, e.g., MART-1 on melanocytes. When targeting those antigens, vitiligo and deafness (Offringa 2009; Johnson et al. 2009a) to a certain degree is frequently observed; uveitis and inner ear toxicity were observed upon adoptive therapy with TCR engineered T cells (Johnson et al. 2009b). Since nearly all tumor-associated antigens are self-antigens, strategies need to be adopted to ensure off-target toxicities are kept to a minimum although toxicity may be a surrogate for clinical efficacy. In the adoptive therapy of melanoma, indeed, off- 
target toxicities generally predict for anti-tumour responses (Overwijk et al. 2003). It is currently under investigation whether T cells with low-avidity TCR are less prone to induce such unwanted side effects. Toxicity, however, can be controlled using steroids to deplete modified T cells locally (Johnson et al. 2009b) or systemically as has been the case in CAR targeting carboanhydrase IX (G250), a renal cell carcinoma antigen also expressed at low levels on bile duct epithelia (Lamers et al. 2006a; Lamers et al. 2006b).

One of the major hurdles of redirected immunotherapy of melanoma is the heterogeneity of antigen expression within the malignant lesions which negatively affects the long-term therapeutic efficacy of the approach. Whereas melanotransferin-positive or HMW-MAApositive melanoma cells may be successfully eliminated by redirected $\mathrm{T}$ cells engineered with the respective CAR, antigen-negative tumor cells, however, will not be recognized by those $\mathrm{T}$ cells. The limitation may be overcome by utilizing mixed $\mathrm{T}$ cell populations modified with different CARs recognizing different antigens of the same tumor. On the other hand, activation of pro-inflammatory immune cells in the tumor microenvironment led to the speculation whether IL-2, secreted in high concentrations by activated T cells, may attract a second wave of non-antigen restricted effector cells which eradicate antigennegative tumor cells. Experiments with antibody-cytokine fusion proteins indicate that at least in an animal model antigen-negative melanoma cells are indeed eliminated when coinoculated with antigen-positive melanoma cells and that the $\mathrm{T}$ cell mediated immune response is followed by a long-lived transferable protective immunity (Becker et al. 1996).

As far as safety concerns, insertional mutagenesis by retro- or lentiviral gene transfer of the TCR or CAR encoding transgene also needs to be addressed. While more than 100 patients have been treated so far, malignant transformation of blood $\mathrm{T}$ cells has not been observed in any case which is in contrast to genetic modification of hematopoietic stem cells. Retrovirally modified, mature polyclonal $\mathrm{T}$ cells seem not to produce clonal amplification upon adoptive transfer (Newrzela et al. 2008). Apart from that, the search for a safer vector system using non-integrating vectors (Perro et al. 2010), RNA transfer (Zhao et al. 2010), or targeted recombination (Carroll 2008) into safe sites is still ongoing. Once adoptively transferred, controlling the engineered $\mathrm{T}$ cell in vivo represents an important option. Novel gene suicide systems using tagged receptor molecules which can be targeted by $\mathrm{T}$ cell depleting antibodies in vivo (Kieback et al. 2008) and inducible caspase-based suicide systems (de Witte et al. 2008) have recently been developed to permit specific depletion of the engineered $\mathrm{T}$ cells rather than total $\mathrm{T}$ cell depletion by steroids.

\section{Targeting the heart of melanoma: do cancer stem cells play a role?}

Current therapeutic strategies in oncology aim to eliminate all cancer cells within a tumor lesion. The histology, however, teaches us that a variety of different cancer cells make up most tumor tissues in addition to non-malignant cells which form the so-called stroma. Transplantation of individual cancer cell subsets from melanoma under limiting dilution conditions revealed that a subset of cancer cells is capable to induce tumors of the same histological phenotype as the parental tumor (Al-Hajj et al. 2003; Schatton et al. 2008; Zabierowski \& Herlyn 2008). Transplantation assays moreover revealed that a low number of sorted cells, most rigorously one cancer cell, is capable to induce tumors when transplanted under appropriate conditions (Quintana et al. 2008). The conclusion drawn from these experiments was that melanoma is organized in a hierarchical manner following an initiator cell or so-called cancer stem cell (CSC). The same observation was drawn from 
other tumor entities (Ailles \& Weissman 2007; Clevers 2011). Low abundance, induction of tumors, self-renewal and differentiation into heterogeneous phenotypes of cancer cells are properties postulated for CSCs. While established as early as 1968 (Fiala 1968), the CSC paradigm experienced revival when Bonnet and Dick (1997) showed that most subtypes of acute myeloid leukemia can be established in their broad diversity by transplantation of a defined and rare subset of malignant $\mathrm{CD}^{+} 4^{+}$cells fully recapitulating the leukemic phenotype (Bonnet \& Dick 1997). A variety of cancer stem cells were subsequently identified in solid tumors including mammary, prostate, pancreatic and colon carcinoma as well as glioma (Al-Hajj et al. 2003; Dalerba et al. 2007; Singh et al. 2003; Ricci-Vitiani et al. 2007; Li et al. 2007). In melanoma, however, the identity of CSCs, their frequency and more even their existence in a given lesion is still controversially debated (Marotta \& Polyak 2009; Rosen \& Jordan 2009; Hill 2006; Shackleton et al. 2009; Shackleton \& Quintana 2010). A first study using the limiting dilution transplantation assay identified a melanoma cell subset which exhibits stem-like capacities and expresses CD20 (Fang et al. 2005). Under more strict assay conditions other groups isolated tumor initiating cells which express either the transporter protein ABCB5 (Schatton et al. 2008) or the nerve growth factor receptor CD271 (Boiko et al. 2010). A recent study from Weissman and colleagues calculated the frequency of tumorigenic cells in melanoma of about 1/2000 cells (Boiko et al. 2010). Investigations by Quintana et al. (Quintana et al. 2010; Quintana et al. 2008) questioned the existence of any melanoma stem cell. Transplantation under more rigorous conditions revealed that nearly every forth randomly chosen melanoma cell $(1 / 2-1 / 15)$ is capable to induce melanoma in the appropriate host and that the potential of melanoma induction is not associated with any surface marker tested so far. This demonstrates that the number of potential CSCs in melanoma may not be necessarily low and that the melanoma inducing capacity is not associated with a fixed phenotype of melanoma cells while these cells reconstitute the exact marker pattern of the parental tumor irrespective of marker positivity or negativity. The observations imply that reversible transformation of the melanoma cell is crucial for the initiation of a new melanoma after xeno-transplantation and that a substantial number of melanoma cells are capable to undergo this transformation process. The melanoma cell may express a variety of different markers at the time of isolation from an established lesion, after transplantation, however, the cell re-programs to the tumor initiating phenotype.

Another so far not appropriately recognized consequence of the CSC paradigm is that a hierarchy of cancer cells is preserved in an established tumor lesion. If the hypothesis is fulfilled for melanoma, some melanoma cells maintain tumor progression whereas the bulk of tumor cells does not. Consequently, specific ablation of the properly rare melanoma sustaining cells, which may but must not be identical to CSCs identified by the transplantation assay, from the established tumor tissue must inevitably lead to a decay of the tumor lesion independently of targeting the cancer cell mass. A most recent study addressed whether specific elimination of defined melanoma cells from an established lesion causes tumor regression (Schmidt et al. 2011). By adoptive transfer of engineered $T$ cells with a CD20 specific CAR established xeno-transplanted tumors could be completely eradicated whereas targeted elimination of any random melanoma cell population did not. The frequency of $\mathrm{CD} 20^{+}$melanoma cells in the tumor tissues was about $1-2 \%$ irrespective of the melanoma subtype; in about $20 \%$ of melanoma samples no CD20+ melanoma cells could be detected. In those tumors, consequently, adoptive transfer of CD20 redirected T cells did not induce tumor regression. Re-expression of CD20 in those tumor cells, moreover, did not reconstitute the capacity to maintain melanoma progression indicating 
that CD20 per se does not induce the capacity. CD20 expression, however, indicates melanoma cells in an established lesion with such tumor maintaining properties. The observations have substantial impact on the future design of melanoma therapy.

First, eradication of hierarchically organized melanoma needs to eliminate those cancer cells which maintain melanoma progression. Elimination of any other cancer cell populations will de-bulk the tumor lesion and induce rapid tumor regression but the tumor will inevitably relapse due to surviving melanoma sustaining cells. The prediction reflects the clinical observation of frequent melanoma relapse after chemotherapy and radiation.

Second, melanoma maintaining cells are rare and unlikely to be eliminated by random targeting as provided by most therapeutic agents. Specific targeting by cytotoxic $\mathrm{T}$ cells redirected towards $\mathrm{CD} 20$ or by CD20-specific therapeutic antibodies like Rituxan ${ }^{\mathrm{TM}}$ (rituximab) or Arzerra ${ }^{\mathrm{TM}}$ (ofatumumab) will be required.

Third, melanoma maintaining cells like other CSCs will less replicate than the majority of cancer cells making anti-proliferative drugs less efficient. Moreover, melanoma CSCs express transporter molecules like ABCB5 (Schatton et al. 2008) which efficiently counteract chemotherapy. Both properties together contribute to CSC resistance towards a variety of physiological and pharmaceutical signals which is frequently described as "dormancy". The dormant state, however, re-activates under so far unknown conditions resulting in melanoma relapse.

Forth, the prevalence of $\mathrm{CD} 20^{+}$melanoma maintaining cells in a tumor lesion may correlate with prognosis. It is unresolved, however, whether those cells accumulate during tumor progression and metastasis.

Fifth, functional and phenotypic plasticity of melanoma maintaining cells may require continuous presence of therapeutic agents specifically targeting those cells which freshly gained melanoma initiating capacities. Clonal evolution of genetic and epigenetic modifications may moreover contribute to cancer cell heterogeneity. In their therapeutic approach, Schmidt and colleagues (2011) used engineered T cells which are known to penetrate tissues, scan for targets and persist for long-term acting as an antigen-specific guardian. This is a major advantage of cellular therapy in comparison to other drugs which need to be present in therapeutic levels over long times which in the case of melanoma may be years. It will therefore be of benefit to de-bulk the tumor mass by conventional therapy in advance to eliminating melanoma initiating cells by specific agents.

\section{Thoughts on future developments}

Current trials using melanoma TILs show first promising results, the efficacy in long-term needs to be confirmed. Using artificially redirected $\mathrm{T}$ cells previous and ongoing phase I studies demonstrate the feasibility in generating suitable numbers of gene-modified $\mathrm{T}$ cells for adoptive transfer. Each trial is being performed with CARs and TCRs which have been empirically optimised and possess subtle but significant differences suggesting that the direct comparison between clinical trial outcomes may not be possible. While lymphodepletion prior $\mathrm{T}$ cell therapy is widely accepted, substitution of IL-2 in high, medium or low doses is still a matter of debate. As such, it is likely that some basic formulations will need to be determined before the field moves on towards larger, multicentre clinical testing of modified T cells.

There are currently clear limitations in adoptive immunotherapy of melanoma and of other malignant diseases. Adoptive immunotherapy uses "drugs" which need to be individually 
generated for each patient. Whilst $\mathrm{T}$ cells can be produced for early phase trials, the ability to generate sufficient cells to perform large scale trials is currently beyond the capabilities of any production centre. With clinical success the biotechnological issues need to be investigated.

\begin{tabular}{|l|l|l|l|l|l|}
\hline $\begin{array}{c}\text { therapeutic } \\
\text { agent }\end{array}$ & $\begin{array}{c}\text { targeted } \\
\text { antigen }\end{array}$ & modification & $\begin{array}{c}\text { clinical } \\
\text { phase }\end{array}$ & principal investigator & $\begin{array}{l}\text { clinicaltrials.gov } \\
\text { or reference }\end{array}$ \\
\hline TIL & unknown & none & II & $\begin{array}{l}\text { Jacob Schachter, Sheba } \\
\text { Medical Center, Tel } \\
\text { Hashomer, Israel }\end{array}$ & NCT00287131 \\
\hline TIL & DMF5 & none & I/II & $\begin{array}{l}\text { Steven A. Rosenberg, } \\
\text { NIH, Bethesda, USA }\end{array}$ & NCT00924001 \\
\hline TIL & unknown & none & I/II & $\begin{array}{l}\text { Brigitte Dreno, CHU de } \\
\text { Nantes, Nantes, France }\end{array}$ & NCT01082887 \\
\hline TIL & unknown & none & I & $\begin{array}{l}\text { Cassian Yee, Fred } \\
\text { Hutchinson Cancer } \\
\text { Center, Seattle, USA }\end{array}$ & NCT00045149 \\
\hline TIL & MART-1 & none & I & $\begin{array}{l}\text { Marcus Butler, Dana } \\
\text { Farber Cancer Institute, } \\
\text { Boston, USA }\end{array}$ & NCT00512889 \\
\hline TIL & unknown & IL-12 & I/II & $\begin{array}{l}\text { Steven A. Rosenberg, } \\
\text { NIH, Bethesda, USA }\end{array}$ & NCT01236573 \\
\hline young TIL & unknown & none & II & $\begin{array}{l}\text { Steven A. Rosenberg, } \\
\text { NIH, Bethesda, USA }\end{array}$ & NCT01118091 \\
\hline young TIL & unknown & none & II & $\begin{array}{l}\text { Steven A. Rosenberg, } \\
\text { NIH, Bethesda, USA }\end{array}$ & NCT00513604 \\
\hline T cells & MART-1 & TCR & I/II & $\begin{array}{l}\text { Brigitte Dreno, CHU de } \\
\text { Nantes, Nantes, France }\end{array}$ & NCT00720031 \\
\hline T cells & MART-1 & TCR & I & $\begin{array}{l}\text { Steven A. Rosenberg, } \\
\text { NIH, Bethesda, USA }\end{array}$ & (Morgan et al. \\
$2006)$
\end{tabular}

Table 1. Clinical trials in the adoptive immunotherapy of melanoma.

As a system eminent limitation the TIL approach is limited by the lack of knowledge of melanoma specificity and the failure to isolate TILs in sufficient numbers from all tumor lesions. The CAR approach, on the other hand, is limited to targets on the cell surface while intracellular antigens cannot be targeted; the TCR approach is limited to proper MHC expression and antigen presentation. While novel strategies are currently explored by combining the advantages and eliminating the limitations, the key aspect for adoptive immunotherapy is that the effector cells need to get to the tumor site and infiltrate the solid 
tumor tissue. In the tumor tissue infiltrating $\mathrm{T}$ cells experience the counter-attack by the immune suppressive environment provided by suppressive cytokines and regulatory cells. Different $\mathrm{T}$ cell subsets may be more effective than others in resisting repression, eliminating regulatory $\mathrm{T}$ cells by lymphodepletion favours therapeutic efficacy of adoptively transferred T cells.

Taken together, strong arguments support the development of cellular immunotherapy of melanoma. Since the initial descriptions of the approach, most of the early technological hurdles in generating melanoma-specific effector cells have been largely overcome and an increasing focus is upon understanding the clinically relevant mechanisms of $\mathrm{T}$ cell directed therapies and how to make the methodology more economic and widely applicable. Adoptive cell therapy in melanoma has moved at a pace and the current clinical and preclinical activities will furthermore determine whether adoptive immunotherapy with redirected $\mathrm{T}$ cells can control melanoma in long-term.

\section{Acknowledgements}

Work in the author's laboratory was funded by a grant from the Deutsche Krebshilfe, Bonn, Germany.

\section{References}

Ailles, L.E. \& Weissman, I.L., 2007. Cancer stem cells in solid tumors. Current Opinion in Biotechnology, 18(5), pp.460-466.

Al-Hajj, M. et al., 2003. Prospective identification of tumorigenic breast cancer cells. Proceedings of the National Academy of Sciences of the United States of America, 100(7), pp.3983-3988.

Atkins, M.B. et al., 1999. High-dose recombinant interleukin 2 therapy for patients with metastatic melanoma: analysis of 270 patients treated between 1985 and 1993. Journal of Clinical Oncology: Official Journal of the American Society of Clinical Oncology, 17(7), pp.2105-2116.

Bargou, R. et al., 2008. Tumor regression in cancer patients by very low doses of a T cellengaging antibody. Science (New York, N.Y.), 321(5891), pp.974-977.

Becker, J.C. et al., 1996. An antibody-interleukin 2 fusion protein overcomes tumor heterogeneity by induction of a cellular immune response. Proceedings of the National Academy of Sciences of the United States of America, 93(15), pp.7826-7831.

Bedikian, A.Y. et al., 2010. Predictive Factors for the Development of Brain Metastasis in Advanced Unresectable Metastatic Melanoma. American Journal of Clinical Oncology. Available at: http://www.ncbi.nlm.nih.gov/pubmed/21150567 [Accessed March 9, 2011]

Bendle, G.M. et al., 2010. Lethal graft-versus-host disease in mouse models of $\mathrm{T}$ cell receptor gene therapy. Nature Medicine, 16(5), pp.565-570, 1p following 570.

Besser, M.J. et al., 2010. Clinical responses in a phase II study using adoptive transfer of short-term cultured tumor infiltration lymphocytes in metastatic melanoma patients. Clinical Cancer Research: An Official Journal of the American Association for Cancer Research, 16(9), pp.2646-2655.

Besser, M.J. et al., 2009. Minimally cultured or selected autologous tumor-infiltrating lymphocytes after a lympho-depleting chemotherapy regimen in metastatic 
melanoma patients. Journal of Immunotherapy (Hagerstown, Md.: 1997), 32(4), pp.415423.

Bluemel, C. et al., 2010a. Epitope distance to the target cell membrane and antigen size determine the potency of $\mathrm{T}$ cell-mediated lysis by BiTE antibodies specific for a large melanoma surface antigen. Cancer Immunology, Immunotherapy: CII, 59(8), pp.1197-1209.

Bluemel, C. et al., 2010b. Epitope distance to the target cell membrane and antigen size determine the potency of $\mathrm{T}$ cell-mediated lysis by BiTE antibodies specific for a large melanoma surface antigen. Cancer Immunology, Immunotherapy: CII, 59(8), pp.1197-1209.

Boiko, A.D. et al., 2010. Human melanoma-initiating cells express neural crest nerve growth factor receptor CD271. Nature, 466(7302), pp.133-137.

Bonnet, D. \& Dick, J.E., 1997. Human acute myeloid leukemia is organized as a hierarchy that originates from a primitive hematopoietic cell. Nature Medicine, 3(7), pp.730737.

Bradbury, P.A. \& Middleton, M.R., 2004. DNA repair pathways in drug resistance in melanoma. Anti-Cancer Drugs, 15(5), pp.421-426.

Bridgeman, J.S. et al., 2010. Building better chimeric antigen receptors for adoptive $\mathrm{T}$ cell therapy. Current Gene Therapy, 10(2), pp.77-90.

Büning, H. et al., 2010. Do CARs need a driver's license? Adoptive cell therapy with chimeric antigen receptor-redirected $\mathrm{T}$ cells has caused serious adverse events. Human Gene Therapy, 21(9), pp.1039-1042.

Burton, A.L. et al., 2011. Prognostic significance of tumor infiltrating lymphocytes in melanoma. The American Surgeon, 77(2), pp.188-192.

Carroll, D., 2008. Progress and prospects: zinc-finger nucleases as gene therapy agents. Gene Therapy, 15(22), pp.1463-1468.

Cheadle, E.J. et al., 2007. Eotaxin-2 and colorectal cancer: a potential target for immune therapy. Clinical Cancer Research: An Official Journal of the American Association for Cancer Research, 13(19), pp.5719-5728.

Chhabra, A., 2010. Mitochondria-centric activation induced cell death of cytolytic T lymphocytes and its implications for cancer immunotherapy. Vaccine, 28(29), pp.4566-4572.

Chmielewski, M., Hombach, A.A. \& Abken, H., 2011. CD28 cosignalling does not affect the activation threshold in a chimeric antigen receptor-redirected T-cell attack. Gene Therapy, 18(1), pp.62-72.

Chmielewski, M. et al., 2004. T cell activation by antibody-like immunoreceptors: increase in affinity of the single-chain fragment domain above threshold does not increase $\mathrm{T}$ cell activation against antigen-positive target cells but decreases selectivity. Journal of Immunology (Baltimore, Md.: 1950), 173(12), pp.7647-7653.

Clark, W.H. et al., 1969. The histogenesis and biologic behavior of primary human malignant melanomas of the skin. Cancer Research, 29(3), pp.705-727.

Clemente, C.G. et al., 1996. Prognostic value of tumor infiltrating lymphocytes in the vertical growth phase of primary cutaneous melanoma. Cancer, 77(7), pp.1303-1310.

Clevers, H., 2011. The cancer stem cell: premises, promises and challenges. Nature Medicine, 17(3), pp.313-319. 
Coccoris, M. et al., 2010. T cell receptor (TCR) gene therapy to treat melanoma: lessons from clinical and preclinical studies. Expert Opinion on Biological Therapy, 10(4), pp.547562.

Coccoris, M. et al., 2008. Long-term functionality of TCR-transduced T cells in vivo. Journal of Immunology (Baltimore, Md.: 1950), 180(10), pp.6536-6543.

Cochlovius, B. et al., 1999. Human melanoma therapy in the SCID mouse: in vivo targeting and reactivation of melanoma-specific cytotoxic $\mathrm{T}$ cells by bi-specific antibody fragments. International Journal of Cancer. Journal International Du Cancer, 81(3), pp.486-493.

Cohen, C.J. et al., 2006. Enhanced antitumor activity of murine-human hybrid T-cell receptor (TCR) in human lymphocytes is associated with improved pairing and TCR/CD3 stability. Cancer Research, 66(17), pp.8878-8886.

Dalerba, P. et al., 2007. Phenotypic characterization of human colorectal cancer stem cells. Proceedings of the National Academy of Sciences of the United States of America, 104(24), pp.10158-10163.

Denninghoff, V.C. et al., 2004. Sentinel lymph node: detection of micrometastases of melanoma in a molecular study. Molecular Diagnosis: A Journal Devoted to the Understanding of Human Disease Through the Clinical Application of Molecular Biology, 8(4), pp.253-258.

Engell-Noerregaard, L. et al., 2009. Review of clinical studies on dendritic cell-based vaccination of patients with malignant melanoma: assessment of correlation between clinical response and vaccine parameters. Cancer Immunology, Immunotherapy: CII, 58(1), pp.1-14.

Eshhar, Z. et al., 1993. Specific activation and targeting of cytotoxic lymphocytes through chimeric single chains consisting of antibody-binding domains and the gamma or zeta subunits of the immunoglobulin and T-cell receptors. Proceedings of the National Academy of Sciences of the United States of America, 90(2), pp.720-724.

Fang, D. et al., 2005. A tumorigenic subpopulation with stem cell properties in melanomas. Cancer Research, 65(20), pp.9328-9337.

Fiala, S., 1968. The cancer cell as a stem cell unable to differentiate. A theory of carcinogenesis. Neoplasma, 15(6), pp.607-622.

Garbe, C. et al., 2010. Diagnosis and treatment of melanoma: European consensus-based interdisciplinary guideline. European Journal of Cancer, 46(2), pp.270-283.

Grimm, E.A. et al., 1982. Lymphokine-activated killer cell phenomenon. Lysis of natural killer-resistant fresh solid tumor cells by interleukin 2-activated autologous human peripheral blood lymphocytes. The Journal of Experimental Medicine, 155(6), pp.18231841.

Grosse-Hovest, L. et al., 1999. Tumor-growth inhibition with bispecific antibody fragments in a syngeneic mouse melanoma model: the role of targeted T-cell co-stimulation via CD28. International Journal of Cancer. Journal International Du Cancer, 80(1), pp.138-144.

Grosse-Hovest, L. et al., 2003. A recombinant bispecific single-chain antibody induces targeted, supra-agonistic CD28-stimulation and tumor cell killing. European Journal of Immunology, 33(5), pp.1334-1340.

Hill, R.P., 2006. Identifying cancer stem cells in solid tumors: case not proven. Cancer Research, 66(4), pp.1891-1895; discussion 1890. 
Hombach, A. \& Abken, H., 2007. Costimulation tunes tumor-specific activation of redirected $\mathrm{T}$ cells in adoptive immunotherapy. Cancer Immunology, Immunotherapy: CII, 56(5), pp.731-737.

Hombach, A.A. et al., 2007. T cell activation by antibody-like immunoreceptors: the position of the binding epitope within the target molecule determines the efficiency of activation of redirected T cells. Journal of Immunology (Baltimore, Md.: 1950), 178(7), pp.4650-4657.

Hong, J.J. et al., 2010. Successful treatment of melanoma brain metastases with adoptive cell therapy. Clinical Cancer Research: An Official Journal of the American Association for Cancer Research, 16(19), pp.4892-4898.

Hughes, M.S. et al., 2005. Transfer of a TCR gene derived from a patient with a marked antitumor response conveys highly active T-cell effector functions. Human Gene Therapy, 16(4), pp.457-472.

Johnson, L.A. et al., 2009a. Gene therapy with human and mouse T-cell receptors mediates cancer regression and targets normal tissues expressing cognate antigen. Blood, 114(3), pp.535-546.

Johnson, L.A. et al., 2009b. Gene therapy with human and mouse T-cell receptors mediates cancer regression and targets normal tissues expressing cognate antigen. Blood, 114(3), pp.535-546.

Kawakami, Y. \& Rosenberg, S.A., 1997. Immunobiology of human melanoma antigens MART-1 and gp100 and their use for immuno-gene therapy. International Reviews of Immunology, 14(2-3), pp.173-192.

Kieback, E. et al., 2008. A safeguard eliminates T cell receptor gene-modified autoreactive T cells after adoptive transfer. Proceedings of the National Academy of Sciences of the United States of America, 105(2), pp.623-628.

Kirkwood, J.M. et al., 2000. High- and low-dose interferon alfa-2b in high-risk melanoma: first analysis of intergroup trial E1690/S9111/C9190. Journal of Clinical Oncology: Official Journal of the American Society of Clinical Oncology, 18(12), pp.2444-2458.

Kirkwood, J.M. et al., 1996. Interferon alfa-2b adjuvant therapy of high-risk resected cutaneous melanoma: the Eastern Cooperative Oncology Group Trial EST 1684. Journal of Clinical Oncology: Official Journal of the American Society of Clinical Oncology, 14(1), pp.7-17.

Kruschinski, A. et al., 2008. Engineering antigen-specific primary human NK cells against HER-2 positive carcinomas. Proceedings of the National Academy of Sciences of the United States of America, 105(45), pp.17481-17486.

Kuball, J. et al., 2007. Facilitating matched pairing and expression of TCR chains introduced into human T cells. Blood, 109(6), pp.2331-2338.

Lamers, C.H.J. et al., 2006. Process validation and clinical evaluation of a protocol to generate gene-modified $\mathrm{T}$ lymphocytes for imunogene therapy for metastatic renal cell carcinoma: GMP-controlled transduction and expansion of patient's $\mathrm{T}$ lymphocytes using a carboxy anhydrase IX-specific scFv transgene. Cytotherapy, 8(6), pp.542-553.

Lamers, C.H.J. et al., 2006. Treatment of metastatic renal cell carcinoma with autologous Tlymphocytes genetically retargeted against carbonic anhydrase IX: first clinical experience. Journal of Clinical Oncology: Official Journal of the American Society of Clinical Oncology, 24(13), pp.e20-22. 
Leiter, U. et al., 2010. Excision guidelines and follow-up strategies in cutaneous melanoma: Facts and controversies. Clinics in Dermatology, 28(3), pp.311-315.

Li, C. et al., 2007. Identification of pancreatic cancer stem cells. Cancer Research, 67(3), pp.1030-1037.

Lo, A.S.Y. et al., 2010. Anti-GD3 chimeric sFv-CD28/T-cell receptor zeta designer T cells for treatment of metastatic melanoma and other neuroectodermal tumors. Clinical Cancer Research: An Official Journal of the American Association for Cancer Research, 16(10), pp.2769-2780.

Marotta, L.L.C. \& Polyak, K., 2009. Cancer stem cells: a model in the making. Current Opinion in Genetics \& Development, 19(1), pp.44-50.

Morgan, R.A. et al., 2003. High efficiency TCR gene transfer into primary human lymphocytes affords avid recognition of melanoma tumor antigen glycoprotein 100 and does not alter the recognition of autologous melanoma antigens. Journal of Immunology (Baltimore, Md.: 1950), 171(6), pp.3287-3295.

Morgan, R.A. et al., 2006. Cancer Regression in Patients After Transfer of Genetically Engineered Lymphocytes. Science, 314(5796), pp.126 -129.

Newrzela, S. et al., 2008. Resistance of mature T cells to oncogene transformation. Blood, 112(6), pp.2278-2286.

Offringa, R., 2009. Antigen choice in adoptive T-cell therapy of cancer. Current Opinion in Immunology, 21(2), pp.190-199.

Overwijk, W.W. et al., 2003. Tumor regression and autoimmunity after reversal of a functionally tolerant state of self-reactive CD8+ T cells. The Journal of Experimental Medicine, 198(4), pp.569-580.

Pak, B.J. et al., 2001. Lineage-specific mechanism of drug and radiation resistance in melanoma mediated by tyrosinase-related protein 2. Cancer Metastasis Reviews, 20(1-2), pp.27-32.

Pak, B.J. et al., 2004. Radiation resistance of human melanoma analysed by retroviral insertional mutagenesis reveals a possible role for dopachrome tautomerase. Oncogene, 23(1), pp.30-38.

Pegram, H.J. et al., 2008. Adoptive transfer of gene-modified primary NK cells can specifically inhibit tumor progression in vivo. Journal of Immunology (Baltimore, Md.: 1950), 181(5), pp.3449-3455.

Perro, M. et al., 2010. Generation of multi-functional antigen-specific human T-cells by lentiviral TCR gene transfer. Gene Therapy, 17(6), pp.721-732.

Pule, M.A. et al., 2008. Virus-specific T cells engineered to coexpress tumor-specific receptors: persistence and antitumor activity in individuals with neuroblastoma. Nature Medicine, 14(11), pp.1264-1270.

Quintana, E. et al., 2010. Phenotypic Heterogeneity among Tumorigenic Melanoma Cells from Patients that Is Reversible and Not Hierarchically Organized. Cancer Cell, 18(5), pp.510-523.

Quintana, E. et al., 2008. Efficient tumour formation by single human melanoma cells. Nature, 456(7222), pp.593-598.

Reinhold, U. et al., 1999. Specific lysis of melanoma cells by receptor grafted T cells is enhanced by anti-idiotypic monoclonal antibodies directed to the scFv domain of the receptor. The Journal of Investigative Dermatology, 112(5), pp.744-750. 
Ricci-Vitiani, L. et al., 2007. Identification and expansion of human colon-cancer-initiating cells. Nature, 445(7123), pp.111-115.

Rivoltini, L. et al., 1995. Induction of tumor-reactive CTL from peripheral blood and tumorinfiltrating lymphocytes of melanoma patients by in vitro stimulation with an immunodominant peptide of the human melanoma antigen MART-1. Journal of Immunology (Baltimore, Md.: 1950), 154(5), pp.2257-2265.

Rivoltini, L. et al., 1996. Binding and presentation of peptides derived from melanoma antigens MART-1 and glycoprotein-100 by HLA-A2 subtypes. Implications for peptide-based immunotherapy. Journal of Immunology (Baltimore, Md.: 1950), 156(10), pp.3882-3891.

Rosen, J.M. \& Jordan, C.T., 2009. The increasing complexity of the cancer stem cell paradigm. Science (New York, N.Y.), 324(5935), pp.1670-1673.

Rosenberg, S.A., 1984. Immunotherapy of cancer by systemic administration of lymphoid cells plus interleukin-2. Journal of Biological Response Modifiers, 3(5), pp.501-511.

Savoldo, B. et al., 2007. Epstein Barr virus specific cytotoxic T lymphocytes expressing the anti-CD30zeta artificial chimeric T-cell receptor for immunotherapy of Hodgkin disease. Blood, 110(7), pp.2620-2630.

Schaft, N. et al., 2003. Peptide fine specificity of anti-glycoprotein 100 CTL is preserved following transfer of engineered TCR alpha beta genes into primary human T lymphocytes. Journal of Immunology (Baltimore, Md.: 1950), 170(4), pp.2186-2194.

Schatton, T. et al., 2008. Identification of cells initiating human melanomas. Nature, 451(7176), pp.345-349.

Schmidt, P. et al., 2011. Eradication of melanomas by targeted elimination of a minor subset of tumor cells. Proceedings of the National Academy of Sciences of the United States of America, 108(6), pp.2474-2479.

Seliger, B., Maeurer, M.J. \& Ferrone, S., 1997. TAP off--tumors on. Immunology Today, 18(6), pp.292-299.

Seliger, B., 2008. Molecular mechanisms of MHC class I abnormalities and APM components in human tumors. Cancer Immunology, Immunotherapy: CII, 57(11), pp.1719-1726.

Shackleton, M. \& Quintana, E., 2010. Progress in understanding melanoma propagation. Molecular Oncology, 4(5), pp.451-457.

Shackleton, M. et al., 2009. Heterogeneity in Cancer: Cancer Stem Cells versus Clonal Evolution. Cell, 138(5), pp.822-829.

Sigalotti, L. et al., 2004. Intratumor heterogeneity of cancer/testis antigens expression in human cutaneous melanoma is methylation-regulated and functionally reverted by 5-aza-2'-deoxycytidine. Cancer Research, 64(24), pp.9167-9171.

Singh, S.K. et al., 2003. Identification of a cancer stem cell in human brain tumors. Cancer Research, 63(18), pp.5821-5828.

Stewart-Jones, G. et al., 2009. Rational development of high-affinity T-cell receptor-like antibodies. Proceedings of the National Academy of Sciences of the United States of America, 106(14), pp.5784-5788.

Vignard, V. et al., 2005. Adoptive transfer of tumor-reactive Melan-A-specific CTL clones in melanoma patients is followed by increased frequencies of additional Melan-Aspecific T cells. Journal of Immunology (Baltimore, Md.: 1950), 175(7), pp.4797-4805. 
Vitale, M. et al., 2005. HLA class I antigen down-regulation in primary ovary carcinoma lesions: association with disease stage. Clinical Cancer Research: An Official Journal of the American Association for Cancer Research, 11(1), pp.67-72.

Waldmann, T.A. \& Tagaya, Y., 1999. The multifaceted regulation of interleukin-15 expression and the role of this cytokine in NK cell differentiation and host response to intracellular pathogens. Annual Review of Immunology, 17, pp.19-49.

Willemsen, R. et al., 2005. Redirecting human CD4+ T lymphocytes to the MHC class Irestricted melanoma antigen MAGE-A1 by TCR alphabeta gene transfer requires CD8alpha. Gene Therapy, 12(2), pp.140-146.

Willemsen, R.A. et al., 2001. A phage display selected fab fragment with MHC class Irestricted specificity for MAGE-A1 allows for retargeting of primary human $\mathrm{T}$ lymphocytes. Gene Therapy, 8(21), pp.1601-1608.

de Witte, M.A. et al., 2008. An inducible caspase 9 safety switch can halt cell therapyinduced autoimmune disease. Journal of Immunology (Baltimore, Md.: 1950), 180(9), pp.6365-6373.

Yvon, E. et al., 2009. Immunotherapy of metastatic melanoma using genetically engineered GD2-specific T cells. Clinical Cancer Research: An Official Journal of the American Association for Cancer Research, 15(18), pp.5852-5860.

Zabierowski, S.E. \& Herlyn, M., 2008. Melanoma stem cells: the dark seed of melanoma. Journal of Clinical Oncology: Official Journal of the American Society of Clinical Oncology, 26(17), pp.2890-2894.

Zhao, Y. et al., 2010. Multiple injections of electroporated autologous T cells expressing a chimeric antigen receptor mediate regression of human disseminated tumor. Cancer Research, 70(22), pp.9053-9061. 


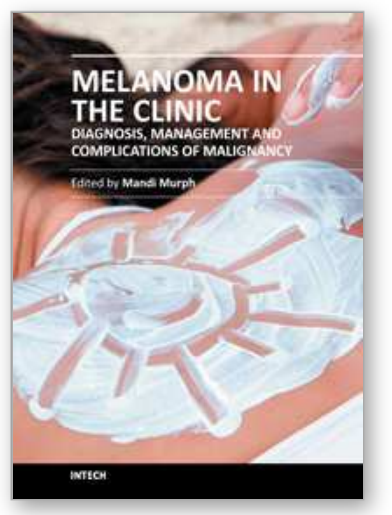

\section{Melanoma in the Clinic - Diagnosis, Management and Complications of Malignancy}

Edited by Prof. Mandi Murph

ISBN 978-953-307-571-6

Hard cover, 310 pages

Publisher InTech

Published online 23, August, 2011

Published in print edition August, 2011

This book provides an excellent overview of how melanoma is treated in the clinic. Since oncologists and clinicians across the globe contributed to this book, each area also explores the unique burdens that geographical areas experience from melanoma subtypes and how these are treated in different settings. It also includes several chapters that illustrate novel methods for diagnosing melanoma in the clinic using new technologies, which are likely to significantly improve outcomes. Several chapters cover surgical techniques and other present very rare or challenging clinical cases of melanoma and how these were treated. The book is geared towards informing clinicians and even patients how melanoma arises, what tools are available and which decisions need to be made by patients and their families in order to treat this devastating disease.

\section{How to reference}

In order to correctly reference this scholarly work, feel free to copy and paste the following:

Patrick Schmidt and Hinrich Abken (2011). Adoptive T-Cell Therapy of Melanoma: Promises and Challenges, Melanoma in the Clinic - Diagnosis, Management and Complications of Malignancy, Prof. Mandi Murph (Ed.), ISBN: 978-953-307-571-6, InTech, Available from: http://www.intechopen.com/books/melanoma-in-the-clinicdiagnosis-management-and-complications-of-malignancy/adoptive-t-cell-therapy-of-melanoma-promises-andchallenges

\section{INTECH}

open science | open minds

\author{
InTech Europe \\ University Campus STeP Ri \\ Slavka Krautzeka 83/A \\ 51000 Rijeka, Croatia \\ Phone: +385 (51) 770447 \\ Fax: +385 (51) 686166 \\ www.intechopen.com
}

\author{
InTech China \\ Unit 405, Office Block, Hotel Equatorial Shanghai \\ No.65, Yan An Road (West), Shanghai, 200040, China \\ 中国上海市延安西路65号上海国际贵都大饭店办公楼405单元 \\ Phone: +86-21-62489820 \\ Fax: +86-21-62489821
}


(C) 2011 The Author(s). Licensee IntechOpen. This chapter is distributed under the terms of the Creative Commons Attribution-NonCommercialShareAlike-3.0 License, which permits use, distribution and reproduction for non-commercial purposes, provided the original is properly cited and derivative works building on this content are distributed under the same license. 